\title{
Naloxone Does Not Alter the Arousal Response Decrement after Repeated Exposure to Hypoxemia during Sleep in Lambs
}

\author{
G. GANESH KONDURI AND JAMES E. FEWELL \\ Department of Pediatrics, Wayne State University School of Medicine, Detroit, Michigan 48201 and \\ Reproductive Medicine Research Group, Departments of Obstetrics and Gynaecology, Medical Physiology and \\ Paediatrics, University of Calgary, Calgary, Alberta T2N 4N1, Canada
}

\begin{abstract}
Experiments were done to determine if endogenous opiates cause the arousal response decrement that follows repeated exposure to hypoxemia during sleep in lambs. Five lambs were anesthetized and instrumented for sleep staging and measurement of arterial $\mathrm{Hb}$ oxygen saturation. No sooner than $\mathbf{3}$ d after surgery, measurements were made in quiet sleep and active sleep during control periods when the lambs were breathing $21 \%$ oxygen and during experimental periods when the lambs were breathing $5 \%$ oxygen. The experimental period was terminated during each epoch by changing the inspired gas mixture back to $21 \%$ oxygen, once the lamb aroused from sleep. After each lamb had been exposed to $5 \%$ oxygen during 100 consecutive epochs of sleep, naloxone-an opiate antagonist-was given i.v. in a dose of $3 \mathrm{mg} / \mathrm{kg}$ as a bolus. The animals continued to be exposed to $5 \%$ oxygen during six more epochs of sleep after the administration of naloxone. Arousal occurred from both sleep states during rapidly developing hypoxemia but was delayed in active sleep compared to quiet sleep. The arterial $\mathrm{Hb}$ oxygen saturation at arousal was significantly lower, and the time to arousal was significantly longer with repeated exposure to hypoxemia during both quiet sleep and active sleep. Naloxone did not alter this arousal response decrement to hypoxemia. Thus, our data provide evidence that endogenous opiates do not play a major role in causing the arousal response decrement that follows repeated exposure to hypoxemia during sleep in lambs. (Pediatr Res 32: 222-225, 1992)
\end{abstract}

Arousal is an important protective response that may prevent severe hypoxemia and death during sleep. Hypoxemia may occur during sleep in individuals with chronic lung disease (1-3). The usual respiratory response to hypoxemia is an increase in ventilation, which tends to increase the alveolar and arterial oxygen tension. However, the ventilatory response to hypoxemia is decreased during active sleep compared to quiet sleep in some species that develop rib cage collapse during active sleep [e.g. lambs (4) and calves (5)]. Thus, the arousal response is an important protective response when we consider the overall cardiorespiratory response to hypoxemia during sleep (6-9).

Received July 29, 1991; accepted March 17, 1992.

Correspondence and reprint requests: James E. Fewell, Ph.D., Heritage Medical Research Building University of Calgary Health Sciences Centre, 3330 Hospital Drive, N.W., Calgary, Alberta T2N 4N1, Canada.

Supported by the Medical Research Council of Canada and done during Dr. Fewell's tenure as an Established Investigator of the American Heart Association and a Heritage Medical Scholar of the Alberta Heritage Foundation for Medical Research.
We have previously shown that repeated exposure to hypoxemia during sleep causes an arousal response decrement in lambs (10), the mechanism of which is not known. However, one possibility is that repeated exposure to hypoxemia increases CNS opiate levels, and this causes the arousal response decrement. Evidence has been provided that endogenous CNS opiate levels are increased in newborn infants (11) and in newborn (12) and adult animals (13) during hypoxemia. Elevated levels of $\beta$ endorphins have also been documented in the cerebrospinal fluid of infants with central apnea (11). Furthermore, endogenous opiates have been shown to play a major role in causing the response decrement after repeated application of a number of stimuli such as pain-evoked potentials in response to repeated stimulation of dental pulp in rabbits (14) and of polysynaptic reflexes in the spinal cord of decerebrate cats in response to peripheral nerve stimulation (15). With this in mind, we have done experiments to test the hypothesis that endogenous opiates play a major role in causing the arousal response decrement to hypoxemia in lambs. We have tested this hypothesis by investigating the effect of naloxone-an opiate receptor antagoniston the arousal response decrement after repeated exposure to hypoxemia in lambs.

\section{MATERIALS AND METHODS}

Experiments were done on five lambs ranging in age from 10 to $19 \mathrm{~d}$. Each lamb was separated from its ewe 1 to $3 \mathrm{~d}$ after birth and was housed in our laboratory in a Plexiglas cage with continuous access to milk (lamb milk replacer, Land O'Lakes, Inc., Fort Dodge, IA). The lambs were among other lambs, fed and slept ad libitum, and soon became accustomed to the surroundings and laboratory personnel.

Surgical preparation. Each lamb underwent one operation before study. Before the operation, each lamb was given atropine sulfate $(0.2 \mathrm{mg} / \mathrm{kg} \mathrm{s.c.})$ and ketamine $\mathrm{HCl}(10 \mathrm{mg} / \mathrm{kg}$ intramuscularly) and its trachea was intubated with a cuffed endotracheal tube. The cuff was inflated to a gas tight fit and anesthesia was maintained by ventilating the lamb's lungs with 1 to $3 \%$ isoflurane (Forane, Anaquest, Madison, WI) in oxygen and nitrous oxide (3:1). An ECG and rectal temperature were monitored during surgery, and core temperature was kept near $39^{\circ} \mathrm{C}$ with a heating pad.

The operation was done between 7 and $16 \mathrm{~d}$ of age when a double-lumen fiberoptic catheter oximeter (model U440 opticath, Oximetrix, Inc., Mountain View, CA) was inserted into the thoracic aorta via a femoral artery for continuous measurement of arterial $\mathrm{Hb}$ oxygen saturation and systemic blood pressure. Electrodes for the following recordings were also implanted: electrocorticogram, electrooculogram, nuchal electromyogram, and diaphragm electromyogram. A reference wire was sutured into the s.c. tissue of the scalp. The electrodes were made in our 
laboratory and were paired, Teflon-coated, multistranded, stainless steel wires (AS 633, Cooner Wire Co., Chatsworth, CA); approximately $3 \mathrm{~mm}$ of the tip of each wire was bared for implantation. The proximal end of each wire was bared and soldered to the appropriate pin of an 18-pin electrical plug that was interfaced with four differential high-impedance probes (7HIP5G, Grass Medical Instruments, Quincy, MA) during a study.

A tracheostomy was also performed and a fenestrated tracheostomy tube (Shiley, Inc., Irvine, CA) placed in the trachea (16). After surgery, the decannulation cannula was inserted into the tracheostomy tube so that airflow during tidal respiration would be through the upper airway. The lambs were allowed to recover from surgery in a Shor-Line intensive care unit for small animals (Schroer Manufacturing Company, Kansas City, MO) and were then placed in a Plexiglas study cage in our sleep laboratory but were not studied before the 3rd postoperative day. The lambs received antibiotics (procaine penicillin G $100000 \mathrm{U} / \mathrm{kg}$; gentamicin sulfate $2 \mathrm{mg} / \mathrm{kg}$ ) for $5 \mathrm{~d}$ beginning on the day of surgery.

Conditions of observations. Our sleep laboratory consists of a large room that contains two sound-attenuating chambers. Each chamber has a one-way viewing mirror as well as a closed circuit video system to observe the lambs. Temperature, sound, and lighting can be precisely controlled in each chamber. Before a study, a partition is placed in the cage to prevent the lamb from turning around once the catheter and electrode are connected. However, the lamb can still lie down, stand up, and feed $a d$ libitum.

For a study, the vascular catheter was connected to a strain gauge manometer (Gould P23ID, Gould, Inc., Oxnard, CA) using rigid pressure monitoring tubing, and the optical connector was connected to the optical module of the oximeter processor. The strain gauge manometer was placed at the approximate level of the heart when the animal was lying down. The inner cannula was placed into the tracheostomy tube and a breathing circuit was attached so that $10 \mathrm{~L} / \mathrm{min}$ of a known gas (i.e. either $21 \%$ oxygen or $5 \%$ oxygen) could be passed by the tracheostomy tube. The 18-pin electrical plug was connected to the differential highimpedance probes; a heavy duty cable connected the differential high-impedance probes to AC preamplifiers (model 7P5 Wide Band AC EEG preamplifier, Grass Instrument Co., Quincy, MA) in the adjacent room. The electrophysiologic signals were highpass filtered using the $1 / 2$ amplitude low-frequency response control on the AC preamplifiers (electrocorticogram $1.0 \mathrm{~Hz}$, electrooculogram $0.3 \mathrm{~Hz}$, and electromyograms $3 \mathrm{~Hz}$ ). The amplified activity from the electrocorticogram was full-wave rectified and integrated (model 7P10 Polygraph Integrator, Grass Instrument Co.) to give a running record of the total accumulated area under the electrocortical waves.

The following electrophysiologic criteria were used to define behavioral state once the animal was lying down. During quiet wakefulness, the electrocorticogram showed a fast-wave, lowvoltage pattern; there were occasional eye movements and there was tonic activity on the nuchal electromyogram. During quiet sleep, the electrocorticogram showed a slow-wave, high-voltage pattern; there were no eye movements and there was tonic activity on the nuchal electromyogram. During active sleep, the electrocorticogram showed a fast-wave, low-voltage pattern; there were rapid eye movements on the electrooculogram, there was no activity on the nuchal electromyogram, and there were occasional fast ear, facial, and limb twitches. Each lamb was allowed to cycle through at least one epoch of quiet sleep before the experiment actually began so that we could determine the amplitude of the integrated electrocortical activity and set strict criteria for defining quiet sleep.

The following electrophysiologic criteria were used to define arousal from sleep. During quiet sleep, the point of arousal was determined by a change in the electrocorticogram from a highvoltage, slow-wave pattern to a low-voltage, fast-wave pattern with continued tonic activity on the nuchal electromyogram.
During active sleep, the point of arousal was determined by a return of tonic activity on the nuchal electromyogram with continued low-voltage, fast-wave activity on the electrocorticogram.

Experimental protocol. During a study, systemic blood pressure, arterial $\mathrm{Hb}$ oxygen saturation, and electrophysiologic signals were recorded on a Grass model 7 polygraph (Grass Instrument Co.) and the lambs were monitored on a closed circuit video system. The lambs were exposed to rapidly developing hypoxemia each time they went to sleep for approximately 100 consecutive epochs ( 29 to $38 \mathrm{~h}$ ). Naloxone was then given i.v. as a bolus in a dose of $3 \mathrm{mg} / \mathrm{kg}$; this dose was chosen because it is 3 to 10 times that used to block endogenous opiate receptors in adult patients (17) as well as in rabbits (14) and fetal sheep (18). The animals were then exposed to hypoxemia during six more epochs of sleep. For each epoch, measurements were made during a 30 -s control period of normal tidal respiration and during an experimental period of rapidly developing hypoxemia. Hypoxemia was terminated during an experimental period by changing the inspired gas mixture to $21 \%$ oxygen once the animal aroused from sleep. Control measurements were made approximately 2 min after the lamb entered quiet sleep and $30 \mathrm{~s}$ after the lamb entered active sleep.

Statistical analysis. For every animal, we determined an average value for each variable during the control period and during the experimental period immediately preceding arousal (heart rate and blood pressure: five cardiac cycles; respiratory frequency: five respiratory cycles) for quiet sleep and active sleep. To analyze the data statistically, we performed a one-factor analysis of variance for repeated measures of the same variable to determine if order (epochs 1-6, 95-100, 101-106) affected the control cardiorespiratory variables or their change before arousal (19). If there were significant differences, a Duncan's multiple range test was done to determine which means were statistically significant at the 0.05 level of significance (20).

\section{RESULTS}

The time to arousal was longer and the arterial $\mathrm{Hb}$ oxygen saturation at arousal was lower in both sleep states after repeated exposure to rapidly developing hypoxemia (Table 1). Naloxone did not alter this arousal response decrement.

Heart rate increased during hypoxemia before arousal in quiet sleep, whereas it decreased during hypoxemia before arousal in active sleep. Naloxone significantly accentuated the decrease in heart rate during hypoxemia in active sleep. Control heart rate was decreased in quiet sleep but not in active sleep after administration of naloxone. Systolic blood pressure increased during hypoxemia before arousal after repeated exposure to hypoxemia in both sleep states; this change was not influenced by administration of naloxone. Respiratory rate increased during hypoxemia before arousal in both sleep states. Naloxone significantly attenuated the ventilatory response to hypoxia in active sleep but not in quiet sleep.

\section{DISCUSSION}

Our experiments provide new and important data about factors that may or may not influence the arousal response from sleep after repeated exposure to a stimulus in young animals. We have provided evidence that endogenous opiates do not play a significant role in causing the arousal response decrement after repeated exposure to hypoxemia in young lambs.

As we have previously reported (10), the time to arousal was longer and the arterial $\mathrm{Hb}$ oxygen saturation at arousal was lower after the lambs were repeatedly exposed to hypoxemia in both active and quiet sleep. The animals continued to show an arousal response decrement after they were given naloxone. Previous experiments provide evidence that the dose of naloxone that we used (i.e. $3 \mathrm{mg} / \mathrm{kg}$ ) was sufficient to block endogenous opiate 
Table 1. Influence of naloxone on arousal response decrement and cardiopulmonary responses after repeated exposure to hypoxemia during sleep in lambs*

\begin{tabular}{|c|c|c|c|c|c|c|}
\hline \multirow[b]{2}{*}{ Variable } & \multicolumn{3}{|c|}{ Quiet sleep } & \multicolumn{3}{|c|}{ Active sleep } \\
\hline & Epochs 1-6 & Epochs 95-100 & Epochs 101-106 & Epochs 1-6 & Epochs $95-100$ & Epochs $101-106$ \\
\hline Time to arousal (s) & $17 \pm 4$ & $32 \pm 12 \dagger$ & $35 \pm 18 \dagger$ & $36 \pm 18$ & $72 \pm 16 \dagger$ & $69 \pm 18 \dagger$ \\
\hline \multicolumn{7}{|l|}{$\mathrm{SaO}_{2}(\%) \ddagger$} \\
\hline Control & $93 \pm 1$ & $92 \pm 1$ & $90 \pm 2$ & $94 \pm 3$ & $92 \pm 3$ & $91 \pm 2$ \\
\hline Change at arousal & $-17 \pm 8$ & $-40 \pm 10 \dagger$ & $-43 \pm 10 \dagger$ & $-37 \pm 17$ & $-52 \pm 12 \dagger$ & $-56 \pm 9 \dagger$ \\
\hline \multicolumn{7}{|l|}{ Heart rate $(\mathrm{bpm})$} \\
\hline Control & $165 \pm 20$ & $170 \pm 14$ & $159 \pm 15 \S$ & $151 \pm 20$ & $158 \pm 20$ & $157 \pm 12$ \\
\hline Change at arousal & $12 \pm 4$ & $24 \pm 6 \dagger$ & $18 \pm 8 \dagger$ & $-5 \pm 3$ & $-7 \pm 3$ & $-35 \pm 10+\S$ \\
\hline \multicolumn{7}{|c|}{ Systolic pressure (mm Hg) } \\
\hline Control & $89 \pm 11$ & $86 \pm 12$ & $88 \pm 14$ & $85 \pm 12$ & $79 \pm 10$ & $87 \pm 15$ \\
\hline Change at arousal & $5 \pm 3$ & $12 \pm 4 \dagger$ & $16 \pm 6 \dagger$ & $8 \pm 4$ & $25 \pm 6 \dagger$ & $22 \pm 9 \dagger$ \\
\hline \multicolumn{7}{|c|}{ Respiratory rate (breaths/min) } \\
\hline Control & $26 \pm 5$ & $31 \pm 5$ & $29 \pm 6$ & $33 \pm 4$ & $35 \pm 9$ & $45 \pm 20$ \\
\hline Change at arousal & $16 \pm 4$ & $22 \pm 4$ & $18 \pm 2$ & $15 \pm 4$ & $19 \pm 4$ & $9 \pm 4+\S$ \\
\hline
\end{tabular}

* Values are means $\pm 1 \mathrm{SD}$ for $n=5$.

$\dagger$ Significant differences by analysis of variance and Duncan's multiple range tests for epochs 1-6 vs epochs 95-100 and/or epochs 101-106.

$\ddagger \mathrm{SaO}_{2}$, arterial oxygen saturation.

$\S$ Significant differences for epochs $95-100$ vs epochs 101-106. Epochs 101-106 were obtained after the animal received 3 mg/kg of naloxone i.v.

receptors. For example, Moss and Scarpelli (21) have shown that a dose of $0.025 \mathrm{mg} / \mathrm{kg}$ naloxone reverses the effects of $\beta$-endorphins in anesthetized dogs and Cianchetti et al. (22) have shown that naloxone induces sleep pattern abnormalities in adults in a dose of 0.15 to $0.20 \mathrm{mg} / \mathrm{kg}$. Furthermore, Janicki et al. (14) reported that naloxone in a dose of $1 \mathrm{mg} / \mathrm{kg}$ prevented habituation of pain-evoked potentials in response to repeated stimulation of dental pulp in rabbits. The dose that we used was higher than that reported in these studies. We therefore believe that it is unlikely that our results could be due to an incomplete block of the opiate receptors. Furthermore, the duration of action of parenterally administered naloxone is between 1 and $4 \mathrm{~h}$ in adults (23) and several hours in neonates, the estimated plasma

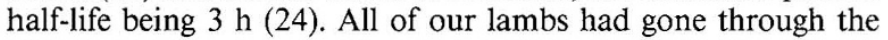
additional six epochs of sleep in less than $3 \mathrm{~h}$ after the administration of naloxone.

Previous investigations have provided evidence that endogenous CNS opiate levels are increased in newborn infants (11) and in newborn (12) and adult animals (13) during hypoxemia. Elevated levels of $\beta$-endorphins have also been documented in the cerebrospinal fluid of infants with central apnea (11). Furthermore, endogenous opiates have been shown to play a major role in causing the response decrement after repeated application of a number of stimuli such as pain-evoked potentials in response to repeated stimulation of dental pulp in rabbits (14), of polysynaptic reflexes in the spinal cord of decerebrate cats in response to peripheral nerve stimulation (15), and of evoked potentials recorded from the reticular formation of the brain in frogs in response to peripheral nerve stimulation (25). These data taken together with the evidence for accumulation of opiates during hypoxia in young animals suggested that endogenous opiates may be responsible for causing the arousal response decrement after repeated exposure to hypoxemia during sleep. However, our data do not support this contention and suggest that alternate mechanisms must be considered.

We do not believe that the arousal response decrement after repeated exposure to hypoxemia is due to sleep fragmentation per se, because sleep fragmentation, produced by intense auditory stimuli over a 36- to 42-h time period, does not significantly affect the arousal response from quiet sleep or active sleep to upper airway obstruction in lambs (16). Response decrement after repeated stimulation can occur through a number of mechanisms including sensory adaptation, nerve accommodation, effector fatigue, and habituation (26).

Because the arousal response from sleep during rapidly developing hypoxemia is initiated by the peripheral chemoreceptors
(27), it is possible that a resetting of these receptors occurs after multiple episodes of hypoxemia (i.e. sensory adaptation) and that this is responsible for the arousal response decrement. However, data from experiments on adult cats provide evidence that prolonged exposure to arterial hypoxemia augments chemosensory activity rather than diminishes it $(28,29)$. We are unaware of similar data in newborns where chemoreceptor activity has been assessed directly by measurements of carotid body discharge rate after chronic or intermittent hypoxemia. However, our cardiovascular and respiratory data would allow one to suggest that the peripheral chemoreceptor response to hypoxemia is not attenuated after repeated exposure to rapidly developing hypoxemia.

It also seems unlikely that the response decrement is due to effector fatigue because a novel stimulus, such as opening the door of the study chamber, would readily arouse the lambs before and after repeated exposure to rapidly developing hypoxemia. At present, we would suggest that the response decrement observed during sleep in response to repeated exposure to hypoxemia is best explained as being due to habituation of the arousal response to a specific stimulus (i.e. chemoreceptor stimulation by hypoxemia). The specific site(s) in the CNS as well as the specific mechanism(s) responsible still remain unknown.

Experiments on anesthetized adult and newborn animals have provided evidence that the primary cardiovascular response to carotid chemoreceptor stimulation is one of bradycardia and systemic vasoconstriction $(30,31)$. However, carotid chemoreceptor stimulation also increases ventilation and this produces secondary changes that consist of tachycardia and systemic vasodilation. We did observe an increase in respiratory rate and diaphragm electrical activity during hypoxemia in both sleep states (Table 1); however, the heart rate response to hypoxemia was influenced by sleep state. As we have previously observed (10), heart rate increased during rapidly developing hypoxemia before arousal in quiet sleep, whereas it decreased during rapidly developing hypoxemia before arousal in active sleep. We would speculate that this results from the influence of sleep state on the CNS processing of information from the periphery during rapidly developing hypoxemia. During quiet sleep, it would appear that information from the periphery is processed such that pulmonary stretch receptor activity dominates and the secondary response of tachycardia is observed, whereas during active sleep it would appear that information from the periphery is processed such that activity from the chemoreceptors prevails and a primary response of bradycardia is observed. The response may depend upon the extent of chemoreceptor stimulation inasmuch as we 
have previously found that inhalation of $10 \%$ oxygen results in tachycardia during both quiet sleep and active sleep in lambs (32).

In our experiments, naloxone caused an alteration in the cardiopulmonary response to hypoxia during active sleep. The decrease in heart rate at arousal was accentuated and the increase in respiratory rate at arousal was attenuated in active sleep during hypoxia after administration of naloxone. We believe that this accentuated decrease in heart rate may have been secondary to the attenuated ventilatory response. A decrease in the ventilatory response to alveolar hypoxia has previously been shown to result in a decrease in the heart rate response in lambs (31). Our findings differ from those of previous studies that reported an increase in the ventilatory response to hypoxia after naloxone in rats (33), newborn infants (34), and newborn rabbits (35). However, these studies were done in animals or infants during wakefulness, and the ventilatory response to hypoxia may be influenced by behavioral state. Mauser and Chapman (36) have shown that naltrexone-an opiate antagonist-attenuates the ventilatory response to hypoxia in pentobarbitone anesthetized rats. Possible reasons for our findings with naloxone may be related to a pharmacologic effect of naloxone independent of its opiate antagonism (37), or endogenous opiates may actually facilitate the ventilatory response to hypoxia during active sleep. Naloxone has been shown to have partial agonist activity at opiate receptors involving behavioral responses in pigeons (37). Furthermore, naloxone in large doses $(>1 \mathrm{mg} / \mathrm{kg})$ has been reported to act as an antagonist of dopaminergic and GABAergic systems (37), though these effects have not been reported in lambs. At present, the state-specific alteration in the cardiopulmonary response to hypoxia during active sleep remains unexplained. This requires further investigation.

The arousal response from sleep has been suggested to be an important protective response that may prevent severe hypoxemia and death during an apneic episode (38). Our data allow us to suggest that if an infant is repeatedly exposed to hypoxemia, either through apneic episodes or abnormalities in gas exchange, the arousal response might be impaired. Our results also indicate that naloxone is unlikely to improve the abnormalities in arousal response to hypoxemia caused by repeated exposure to hypoxemia during sleep.

\section{REFERENCES}

1. Muller NL, Francis PW, Gurwitz D, Levison H, Bryan AC 1980 Mechanism of hemoglobin desaturation during rapid-eye-movement sleep in normal subjects and in patients with cystic fibrosis. Am Rev Respir Dis 121:463469

2. Taussig LM 1986 Long-term management and pulmonary prognosis in bronchopulmonary dysplasia. In: Farrell PM, Taussig LM (eds) Bronchopulmonary Dysplasia and Related Chronic Disorders. Ross Laboratories, Columbus, OH, pp 126-135

3. Hudgel DW, Kellum R, Martin RJ, Johnson B 1982 Depressed arousal response to airflow obstruction: a possible factor in near fatal nocturnal asthma. Am Rev Respir Dis (Suppl) 125:202(abstr)

4. Henderson-Smart DJ, Read DJC 1979 Ventilatory responses to hypoxemia during sleep in the newborn. J Dev Physiol 1:195-208

5. Jeffrey HE, Read DJC 1980 Ventilatory responses of newborn calves to progressive hypoxia in quiet and active sleep. J Appl Physiol 48:892-895

6. Gastaut H, Tassinari CA, Duron B 1966 Polygraphic study of the episodic diurnal and nocturnal (hypnic and respiratory) manifestations of the Pickwickian syndrome. Brain Res 2:167-186

7. Walsh RE, Michealson ED, Harkerload LE, Zichelboim A, Sackner MA 1972 Upper airway obstruction in obese patients with sleep disturbances and somnolence. Ann Intern Med 76:185-192

8. Remmers JE, DeGroot WJ, Sauerland EK, Anch AM 1978 Pathogenesis of upper airway occlusion during sleep. J Appl Physiol 44:931-938

9. Frank Y, Kravath RE, Pollak CF, Weitzman ED 1983 Obstructive sleep apnea and its therapy: clinical and polysomnographic manifestations. Pediatrics 71:737-740

10. Fewell JE, Konduri GG 1989 Influence of repeated exposure to rapidly developing hypoxemia on the arousal and cardiopulmonary response to rapidly developing hypoxemia in lambs. J Dev Physiol 11:77-82

11. Orlowski JP 1986 Cerebrospinal fluid endorphins and the infant apnea syndrome. Pediatrics 78:233-237

12. Moss IR, Inman JG 1989 Proopiomelanocortin opioids in brain, CSF, and plasma of piglets during hypoxia. J Appl Physiol 66:2280-2286

13. Schaeffer JI, Haddad GG 1988 Ventilatory response to moderate and severe hypoxia in adult dogs: role of endorphins. J Appl Physiol 65:1383-1388

14. Janicki P, Libich J, Gumalka W 1979 Lack of habituation of pain evoked potentials after naloxone. Pol J Pharmacol Pharm 31:201-205

15. Fernandez-Guardiola A, Pellicer F, Leon-Olea M, Asai M, Sanchez-Alvarez M 1989 Habituation and dehabituation of the spinal polysynaptic reflex responses: modification by naloxone and opiates and their anatomical correlates. Neuropeptides 14:115-120

16. Fewell JE 1987 Influence of short-term sleep fragmentation produced by intense auditory stimuli on the arousal response to upper airway obstruction in lambs. J Dey Physiol 9:409-417

17. Akiyama Y, Nishimura M, Suzuki A, Yamamoto M, Kishi F, Kawakami Y 1990 Naloxone increases ventilatory response to hypercapnic hypoxia in healthy adult humans. Am Rev Respir Dis 142:301-305

18. Moss IR, Scarpelli EM $1984 \mathrm{CO}_{2}$ and naloxone modify sleep/wake state and activate breathing in the acute fetal lamb preparation. Respir Physiol 55:325340

19. Winer BJ 1971 Single-factor experiments having repeated measures on the same elements. In: Statistical Principles in Experimental Design. McGrawHill Book Company, New York, pp 261-308

20. Zar JH 1979 Multiple comparisons. In: Biostatistical Analyses. Prentice-Hall, Englewood Cliffs, NJ, pp 151-162

21. Moss IR, Scarpelli EM $1981 \beta$-endorphin central depression of respiration and circulation. J Appl Physiol 50:1011-1016

22. Cianchetti C, Masala C, Olivari P, Giordano G 1984 Sleep pattern alterations by naloxone. Partial prevention by haloperidol. Psychopharmacol 83:179182

23. Misra AL 1978 Metabolism of opiates. In: Adler ML, Manara L, Samanin R (eds) Factors Affecting the Action of Narcotics. Raven Press, New York, pp 297-343

24. Brice JEH, Moreland TA, Walker CHM 1979 Effects of pethidine and its antagonists on the newborn. Arch Dis Child 54:356-36

25. Boxer MI 1983 The possible role of opiates in habituation of responses recorded in the reticular formation of the frog to peripheral stimulation. Neurosci Lett 40:61-65

26. Sharpless S, Jasper H 1956 Habituation of the arousal reaction. Brain 79:655680

27. Fewell JE, Kondo CS, Dascalu V, Filyk SC 1989 Influence of carotid-denervation on the arousal and cardiopulmonary response to rapidly developing hypoxemia in lambs. Pediatr Res 25:473-477

28. Hornbein TF, Severinghaus JW 1969 Carotid chemoreceptor response to hypoxia and acidosis in cats living at high altitude. J Appl Physiol 27:837839

29. Barnard P, Andronikou S, Pokorski M, Smatresk N, Mokashi A, Lahiri S 1987 Time-dependent effect of hypoxia on carotid body chemosensory function. J Appl Physiol 63:685-691

30. Daly M de B 1972 Interaction of cardiovascular reflexes. In: Gilliand I, Francis J (eds) Lectures on the Scientific Basis of Medicine. The Athlone Press, University of London, London, England, pp 307-332

31. Fewell JE, Ramick RA, Taylor BJ 1986 Influence of the ventilatory response on the cardiovascular response to hypoxemia in lambs. Pediatr Res 20:368A(abstr)

32. Fewell JE, Williams BJ, Hill DE 1984 Sleep state does not affect the cardiovascular response to alveolar hypoxia in lambs. J Dev Physiol 6:401-405

33. Olsen Jr EB 1987 Naloxone accelerates the rate of ventilatory acclimatization to hypoxia in awake rats. Life Sci 41:161-167

34. De Boeck C, Van Reempts P, Rigatto H, Chernick V 1984 Naloxone reduces decrease in ventilation by hypoxia in newborn infants. J Appl Physiol 56:1507-1511

35. Gruenstein MM, Hazinski TA, Schlueter MA 1981 Respiratory control during hypoxia in newborn rabbits: implied action of endorphins. J Appl Physiol $51: 122-130$

36. Mauser PJ, Chapman RW 1987 Role of endogenous opioids on ventilation and chemical control of breathing in pentobarbitone anesthetized rats. Pharmacology 35:317-326

37. Sawynok J, Pinsky C, Labella FS 1979 Minireview on the specificity of naloxone as an opiate antagonist. Life Sci 25:1621-1632

38. Phillipson EA, Sullivan CE 1978 Arousal: the forgotten response to respiratory stimuli. Am Rev Respir Dis 118:807-809 